\title{
Hyperkinesia in ancient China: perspectives and prescriptions
}

\author{
Jia Liu ${ }^{1 *}$ and Lu-ning Wang ${ }^{2 *}$
}

\begin{abstract}
${ }^{1}$ Department of Neurology, Xuanwu Hospital, Capital Medical University, Beijing, China
${ }^{2}$ Department of Geriatric Neurology, Chinese PLA General Hospital, Beijing, China
\end{abstract}

Received 30 May 2016; Accepted 2 July 2016

Key words: Ancient China, hyperkinesia, pathophysiology, therapy.

Hyperkinesia (hyperkinetic movement disorders), a term derived from Greek, is used to describe a state of excessive restlessness featured by a large variety of disorders that affect the ability to control movement, such as Huntington's disease, neuroacanthocytosis, and Tourette's syndrome, as well as the secondary symptom in certain special conditions such as Sydenham's chorea and chorea during pregnancy or after stroke. Actually, any disorders in the basal ganglia can give rise to hyperkinesia, including neurodegenerative, pharmacologic, and metabolic factors. In Western medical history, "chorea" as a representative hyperkinesia was first mentioned as a medical concept by Paracelcus in the 16th century. ${ }^{1}$ Then the first description of childhood chorea by Sydenham was followed in 1686; however, its correlation with rheumatic fever was not understood until the 19th century. ${ }^{2}$ Huntington's chorea was first regarded as an adult-onset, hereditary movement disorder by George Huntington in $1872,{ }^{3}$ while neuroacanthocytosis was first described as Levine-Critchley syndrome based on the reports by Levine and Critchley in 2 families from North America in the 1960s. ${ }^{4}$

In comparison to the Western history of medicine, many records on recognizing and treating hyperkinesia can be detected in the thousands of years of history of traditional Chinese medicine (TCM). For instance, “痚疭 (Chi Zong)" is the TCM term that most resembles hyperkinesia. The earliest record of Chi Zong can be traced back thousands of years. Due to the language barrier, most of the information has not been widely

\footnotetext{
* Address correspondence to: Dr. Jia Liu, Department of Neurology, Xuanwu hospital, Capital Medical University, Changchun Street 45 Beijing 100053, China. (Email: jason_liu1984@163.com)

Dr. Lu-ning Wang, Department of Geriatric Neurology, Chinese PLA General Hospital, Fuxing Road 28, Beijing 100853, China.

(Email: ln_wang301@sohu.com)
}

known by Western scholars. In this editorial, we aim to summarize the main TCM philosophies and treatments of hyperkinesia in ancient China by searching the literature of different eras, and to find possible links to diagnosis and treatment today.

The concept of Chi Zong was first mentioned in Basic Questions. $^{5}$ As we previously described, the Yellow Emperor's Internal Classic is the first Chinese medical text and the origin of TCM theory. It was completed approximately sometime during the Warring States Period and the Han Dynasty (from 475 BC to AD 220). ${ }^{6}$ It is composed of 2 parts, Basic Questions and Spiritual Pivot. In TCM, there are 6 evils of nature: wind, cold, summer heat, humidity, dryness, and fire. They are regarded as pathogens and can attack 5 depots (the liver, the heart, the spleen, the lung, and the kidneys) and 6 palaces. According to visceral manifestation theory (the basic principles of TCM), the heart is usually considered as the official functioning as ruler in the 6 depots and 6 palaces. In Basic Questions, the idea that "Spirit brilliance originates from the heart" was first raised. ${ }^{5,7}$ The author thought Chi Zong was raised by disturbance of evil fire up to the heart. To some extent, it resembles the current pathophysiology of Sydenham's chorea, which is usually caused by rheumatic fever. Interestingly, chorea and rheumatic heart disease are highly correlated with rheumatic fever.

In the Han Dynasty, a famous Chinese physician, Zhang Zhongjing (AD 150-219), who is generally thought of as the sage of Chinese medicine, mentioned in his medical masterpiece Shang Han Za Bing Lun that Chi Zong was a kind of febrile diseases with evil fire up to the heart, as well as liver-wind stirring. ${ }^{8}$ The prescription of Feng Yin decoction was recommended (Table 1). ${ }^{9}$ In the Dang Dynasty, the famous Confucian physician Sun Simiao (AD 581-682) discussed hyperkinesia in the 
TABLE 1. Prescriptions of TCM for hyperkinesia therapy in ancient China

\begin{tabular}{|c|c|c|}
\hline Pathophysiology & Prescriptions & Ingredients \\
\hline $\begin{array}{l}\text { Evil fire up to heart and } \\
\text { liver-wind stirring }\end{array}$ & Feng Yin decoction ${ }^{9}$ & $\begin{array}{l}\text { Rheum palmatum L., Rhizoma zingiberis, Os Draconis, Cinnamomum cassia (L.) J. Presl, Glycyrrhiza uralensis Fisch., } \\
\text { Ostreae, Gypsum rubrum, talcum, Halloysitum rubrum, Halloysitum album, fluorite, and gypsum }\end{array}$ \\
\hline \multirow[t]{2}{*}{$\begin{array}{l}\text { Wind in meridians and } \\
\text { collaterals }\end{array}$} & $\begin{array}{l}\text { San Huang Shi Gao } \\
\text { decoction }^{11}\end{array}$ & $\begin{array}{l}\text { Gypsum, Coptis chinensis Franch., Phellodendron chinense C.K.Schneid., Scutellaria baicalensis Georgi, Gardenia } \\
\text { jasminoides J.Ellis, Ephedra sinica Stapf, lobster sauce, Zingiber officinale Roscoe, Ziziphus jujuba Mill., Camellia } \\
\text { sinensis (L.) Kuntze }\end{array}$ \\
\hline & Da Qin Jiao decoction ${ }^{14}$ & $\begin{array}{l}\text { Gentiana macrophylla Pall., Notopterygium franchetii H. Boissieu, Saposhnikovia divaricata (Turcz.) Schischk., Angelica } \\
\text { dahurica (Hoffm.) Benth. \& Hook.f. ex Franch. \& Sav., Asarum sieboldii Miq., Rehmannia glutinosa (Gaertn.) DC., } \\
\text { Angelica sinensis (Oliv.) Diels, Ligusticum striatum DC., Paeonia lactiflora Pall., Buthus martensii Karsch }\end{array}$ \\
\hline \multirow[t]{2}{*}{$\begin{array}{l}\text { Yin deficiency of liver } \\
\text { and kidney }\end{array}$} & $\begin{array}{l}\text { Da Ding Feng Zhu He Bu } \\
\text { Gan decoction }{ }^{17}\end{array}$ & $\begin{array}{l}\text { Carapax testudinis, Carapax trionycis, colla corii asini, hen egg yolk, Ophiopogon japonicus (Thunb.) Ker Gawl., Paeonia } \\
\text { lactiflora Pall., oyster, Glycyrrhiza uralensis Fisch., Schisandra chinensis (Turcz.) Baill., Cannabis sativa L., Ziziphus } \\
\text { jujuba Mill., Angelica sinensis (Oliv.) Diels, Ligusticum striatum DC., Rehmannia glutinosa (Gaertn.) DC., } \\
\text { Chaenomeles sinensis (Dum.Cours.) Koehne }\end{array}$ \\
\hline & $\begin{array}{l}\text { Zhen Gan Xi Feng } \\
\text { decoction }^{18}\end{array}$ & $\begin{array}{l}\text { Paeonia lactiflora Pall., Scrophularia ningpoensis Hemsl., Asparagus cochinchinensis (Lour.) Merr., Rehmannia } \\
\text { glutinosa (Gaertn.) DC., Os Draconis, oyster, Dendranthema morifolium (Ramat.) Tzvelev, Gastrodia elata Blume, } \\
\text { Uncaria rhynchophylla (Miq.) Miq. ex Havil., Angelica sinensis (Oliv.) Diels }\end{array}$ \\
\hline
\end{tabular}

book Qian Jin Yi Fang. ${ }^{10}$ He cited the theory of Xu Sibo, a famous physician in the Nan-Bei Dynasty (AD 420-589), that the disturbance of Qi in the heart was the primary cause. As an important TCM concept, Qi was believed to be a flowing energy in the body to ensure the free flow of emotions, blood, and water. Liver-wind stirring with mutual interference of heart and liver was also involved in the pathophysiology. Another famous medical book Wai Tai Mi Yao, edited by the physician Wang Tao, is regarded as the collection of the medical essence of the Tang Dynasty and before. In Wai Tai Mi Yao, the prescription "San Huang Shi Gao decoction" (Table 1) was provided, which focused on the wind in meridians and collaterals. ${ }^{11}$

During the Song Dynasty, the important medical book Xiao Er Yao Zheng Zhi Jue" was compiled, which is generally regarded as the earliest Chinese pediatric text. $^{12}$ The knowledge in this book mainly came from the most famous Chinese pediatrician Qian Yi (AD 1032-1117) and was collected by another pediatrician, Yan Xiaozhong. The author vividly described the clinical manifestations of Chi Zong, with head drops, orofacial dyskinesia, facial tics, and shrugging, which are the features of neuroacanthocytosis according to current opinion. ${ }^{13}$ He pointed out that liver-wind stirring with the interference of heart refers to the symptoms of involuntary hyperkinetic movement. The deficiency of Qi in the spleen with phlegm, a harmful liquid substance in the body, was also involved. Thereafter, "Da Qin Jiao decoction" (Table 1) was noted by Liu Wansu (AD 11181200) in the book Su Wen Bing Ji Qi Yi Bao Ming Ji for the treatment of hyperkinesia with regard to the wind in meridians and collaterals. ${ }^{14}$ In the Ming Dynasty, Chi Zong was described by Zhang Jingyue (AD 1563-1640) in the book Jing Yue Quan Shu. ${ }^{15}$ He observed that people with severe illness or postpartum hemorrhage were prone to suffer from hyperkinesia. Regarding postpartum hemorrhage, he believed the mechanism to be that the essence and the blood in the body were lost during the pregnancy, which further led to dystrophy of the tendons and vessels, as well as wind shaking. As a current concept of disease, chorea gravidarum is a good example explained by this point of view.

In the Qing Dynasty, the role of the kidneys in the pathophysiology of Chi Zong was emphasized. According to visceral manifestation theory, the kidneys ruled the body's bones and stored mind. Furthermore, the kidneys were acknowledged as the congenital foundation of the human body, and Yin deficiency in the kidneys could further lead to Yin deficiency in the liver. ${ }^{16}$ The concept of Yin-Yang (dualism) is an important theory in TCM. If the balance of Yin-Yang was broken due to Yin deficiency, this would lead to the symptoms. Actually, the prescriptions of $\mathrm{Da}$ Ding Feng Zhu $\mathrm{He} \mathrm{Bu}$ Gan decoction (Table 1$)^{17}$ in the book Wen Bing Tiao Bian and Zhen Gan Xi Feng decoction (Table 1$)^{18}$ in the book were both focused on Yin deficiency in kidney and liver. The clinical heterogeneity of the concept Chi Zong should be noticed. For instance, the cases that happened during childhood could not be completely distinguished from seizures. In comparison to tonus, the concept of Chi Zong was formally specified as hyperkinesia in the Qing dynasty in the book Wen Bing Tiao Bian, written by Wu Tang (1758-1836). ${ }^{17}$ Actually, the classification of diseases in TCM and modern medicine is quite different. The clinical manifestation is the key point in TCM, rather than etiology. Therefore, different diseases that share the same symptoms can receive the same treatments in TCM.

In summary, the concept of Chi Zong was first mentioned in the Yellow Emperor's Internal Classic, which is the first Chinese medical classic and the origin of TCM theory. The pathogenesis of Chi Zong was believed to be a disturbance of evil fire up to the heart. During the Han and Tang dynasties, liver-wind stirring 
with mutual interference of the heart was considered as the main cause. In the Song and Ming dynasties, the deficiency of Qi in the spleen with phlegm, and the loss of essence and blood in the body were raised as the additional mechanisms. In the Qing dynasty, Yin deficiency in the kidneys was thought to be the source, because the kidneys were acknowledged as the congenital foundation of the human body. The Chinese medical formulae were produced based on the therapeutic targets. Nowadays, genetic testing has been widely used to diagnose the hereditary diseases, such as Huntington's disease and neuroacanthocytosis. Therefore, the diseases in the category of hyperkinesia can be accurately classified. With regard to treatment today, most of the ingredients in the prescriptions are still considered as complementary for hyperkinesia. However, the efficacy and safety evidence from clinical trials is required.

\section{Disclosures}

The authors do not have anything to disclose.

\section{REFERENCES:}

1. Lanska DJ. Chapter 33: the history of movement disorders. Handbook of Clinical Neurology. 2010; 95: 501-546.

2. Walker K, Lawrenson J, Wilmshurst JM. Sydenham's choreaclinical and therapeutic update 320 years down the line. $S$ Afr MedJ. 2006; 96(9 Pt 2): 906-912.
3. Lanska DJ. George Huntington (1850-1916) and hereditary chorea. J Hist Neurosci. 2000; 9(1): 76-89.

4. Liu J, Bader B, Danek A. Neuroacanthocytosis in China: a review of published reports. Tremor Other Hyperkinet Mov (N Y). 2014; 4: 248.

5. Yellow Emperor. Yellow Emperor's Internal Classic: Plain Questions. Beijing, China: People's Medical Publishing House; 1963.

6. Liu J, Wang LN, Tian JZ. Recognition of dementia in ancient China. Neurobiol Aging. 2012; 33(12): 2948.e11-13.

7. Unschuld PU. Huang Di Nei Jing Su Wen: Nature, Knowledge, Imagery in an Ancient Chinese Medical Text. Berkeley, CA: University of California Press; 2003.

8. Zhang ZJ. Shang Han Lun. Chongqing, China: Chongqing People's Publishing House; 1956.

9. Zhang ZJ. Jin Gui Yao Lue. Beijing, China: Academy Press; 2008

10. Sun SM. Qian Jin Yi Fang. Beijing, China: People's Medical Publishing House; 1955.

11. Wang T. Wai Tai Mi Yao. Beijing, China: HuaXia Publishing House; 1993.

12. Qian Y. Xiao Er Yao Zheng Zhi Jue. Beijing, China: People's Medical Publishing House; 2006.

13. Schneider SA, Lang AE, Moro E, et al. Characteristic head drops and axial extension in advanced chorea-acanthocytosis. Mov Disord. 2010; 25(10): 1487-1491.

14. Liu WS. Su Wen Bing Ji Qi Yi Bao Ming Ji. Beijing, China: People's Medical Publishing House; 2005.

15. Zhang JY. Jing Yue Quan Shu. Beijing, China: People's Medical Publishing House; 1991.

16. Li ZZ. Yi Zong Bi Du. Tianjin, China: Tianjin Science and Technology Press; 2012.

17. Wu T. Wen Bing Tiao Bian. Fujian, China: Fujian Science and Technology Press; 2010.

18. Zhang XC. Yi Xue Zhong Zhong Can Xi Lu. Shanxi, China: Shanxi Science and Technology Press; 2009. 